\title{
Risk Factors for Lymph Node Metastasis of Soft Tissue Sarcomas of the Head, Neck and Extremities, And the Clinical Significance of Negative Lymph Node Dissection
}

\section{Qikun Liu}

Tongji Hospital of Tongji Medical College of Huazhong University of Science and Technology

\section{Xiaojun Yu}

Tongji Hospital of Tongji Medical College of Huazhong University of Science and Technology

\section{Mengwei Li}

Tongji Hospital of Tongji Medical College of Huazhong University of Science and Technology

\section{Zhiwei Li}

Tongji Hospital of Tongji Medical College of Huazhong University of Science and Technology

\section{Yongqiao Jiang}

Tongji Hospital of Tongji Medical College of Huazhong University of Science and Technology

Hao Kang ( $\sim$ kanghao100@vip.sina.com)

Tongji Hospital of Tongji Medical College of Huazhong University of Science and Technology https://orcid.org/0000-0002-6582-2895

\section{Research}

Keywords: Soft tissue sarcoma, lymph node metastasis, risk factors, prognosis, negative lymph node dissection

Posted Date: August 17th, 2021

DOI: https://doi.org/10.21203/rs.3.rs-786630/v1

License: (c) (i) This work is licensed under a Creative Commons Attribution 4.0 International License. Read Full License

Version of Record: A version of this preprint was published at Journal of Orthopaedic Surgery and Research on March 18th, 2022. See the published version at https://doi.org/10.1186/s13018-022-030503. 


\section{Abstract}

Background: To determine the risk factors for lymph node metastasis (LNM) of soft tissue sarcomas (STS) of the head, neck, and extremities, and the clinical outcome of negative lymph node dissection (NLND).

Methods: We pooled patients of STS using the Surveillance, Epidemiology, and End Results (SEER) database from 1988 to 2015. Logistics regression analysis to identify risk factors for LNM, the Cox proportional hazards model and Fine-Grey's model were used for survival analysis, Propensity score matching analysis (PSM) was further used to clarify the impact of NLND on patient prognosis.

Results: A total of 3,276 patients were enrolled in our study, of whom 283 (8.6\%) developed LNM. Rhabdomyosarcoma had the highest rate of LNM (25.3\%), followed by clear cell sarcoma $(16.8 \%)$ and epithelioid sarcoma (12.4\%), while leiomyosarcoma had the lowest rate of LNM (1.3\%). Sex, tumor size, grade, histology, and site were significantly associated with LNM. Age, tumor size, grade, stage, histology, and marital status were independent prognostic factors for the cancer-specific survival for patients without LNM. For specific histologic subtypes of STS, NLND significantly improves overall survival (HR: $0.718,95 \% \mathrm{Cl}, 0.535-0.962 ; \mathrm{P}=0.026)$ and cancer-specific survival (HR: 0.699, 95\% Cl, 0.506-0.967; $P=0.031$ ) and reduces cancer-specific mortality (Gray's test, $P=0.017)$. However, for patients with leiomyosarcoma, NLND did not improve overall survival $(P=0.46)$ or reduce cancer-specific mortality (Gray's test, $\mathrm{P}=0.772$ ).

Conclusions: We identified the rate of LNM and risk factors for LNM in STS of the head, neck and extremities. In addition, prophylactic NLND treatment is necessary and has a clinical benefit for patients with STS who are at high risk for LNM, but has no significant impact on the prognosis of patients with leiomyosarcoma.

\section{Introduction}

Soft tissue sarcomas (STS) are rare heterogeneous solid tumors of mesenchymal cell origin, more than 50 different histologic subtypes of STS have been identified to date[1]. Common subtypes of STS include malignant fibrous histiocytoma, liposarcoma, and leiomyosarcoma. And extremities (30.7\%), truncal or visceral locations (50.4\%), retroperitoneum $(11.7 \%)$, and head or neck $(7.2 \%)$ are the most common primary sites[2]. These mesenchymal tumors have a propensity for hematogenous metastasis, distant metastasis rates ranged from $12-37.7 \%[3,4]$. The common metastatic sites were lung and bone $[4,5]$. However, lymph node metastasis (LNM) is relatively rare in most STS[6]. It is reported that the incidence of LNM ranged from $0.9-6 \%[3,7-10]$. The rate of LNM varies greatly in different sarcomas. $A$ retrospective study by Keung et al. involving 89,870 extremity/trunk STS found that small cell sarcoma (19\%), clear cell sarcoma (16\%), epithelioid (13\%), and angiosarcoma (6\%) as the subtypes with the highest incidence of LNM[8]. The study of Sawamura et al. showed that the STS with the highest rate of LNM were clear cell sarcoma (38\%), rhabdomyosarcoma (37\%), epithelioid sarcoma (30\%), 
angiosarcoma (20\%), and Ewing's sarcoma of soft tissue (16\%)[9]. Some studies have classified clear cell sarcoma, rhabdomyosarcoma, epithelioid sarcoma, and angiosarcoma as subtypes with a high risk of LNM[11]. Based on the results of previous studies, we collected six subtypes STS with a high risk of LNM for analysis.

It is generally believed that LNM is associated with a poorer prognosis[3, 10,11]. Crettenand et al. found that LNM of STS hurts both overall (median survival: 15.1 months vs 73.9 months, respectively; $p=$ 0.002 ) and disease-free survival (median disease-free survival: 8.0 months vs 33.0 months, respectively; $p=0.006$ )[12]. And the 8th edition of the AJCC staging system defines lymph node involvement as being stage IV disease for sarcomas of the trunk and extremities. Therefore, identifying which patients tend to develop LNM and the risk factors for LNM is important for clinician decision making and patient prognosis. However, identifying these patients has always been a challenge. Furthermore, the prognostic factors of patients with STS without LNM who have undergone surgical treatment need further clarification. At present, there are no studies on the prognostic impact of NLND treatment on patients with STS of the head, neck and extremities.

Given the above considerations, we conducted this study. The purpose of our study was to identify the risk factors for LNM in patients with six histological subtypes of STS, the prognostic factors of patients without LNM and the impact of NLND treatment on patients' prognosis.

\section{Material And Methods}

\section{Data source and patient selection}

Clinical data for this retrospective study were obtained from the Surveillance Epidemiology and End Results (SEER) database. The SEER database is free and publicly accessible, and individual consent for this retrospective analysis was waived as the patient information is anonymous. The study was conducted following the Declaration of Helsinki (as revised in 2013). Data of all patients were downloaded using the SEER ${ }^{*}$ Stat software (version 8.3.6). The final population of our study was selected based on the following inclusion criteria: 1).Diagnosis by positive pathology; 2).Tumor sites: head, neck and extremities; 3).Histological codes: 8890-1, 8893-6, 8900-8902, 8910, 8912, 8920, 9120, 9130, 9133, $9150,9170,9260,9364,9473,8804,8005,9044 ; 4)$. The tumor was the first primary tumor at the diagnosis; 5). Know lymph node status and survival month. The detailed inclusion and exclusion process is shown in Fig. 1.

\section{Variables and outcomes}

In our study, we collected the patient's demographic variables: age, sex, race, marital status, year of diagnosis; oncological variables: tumor size, site, laterality, stage, grade, lymph node status, histology; therapeutic variables: surgery, lymph node dissection. According to previous studies, STS have different oncological features in different age groups of patients. Age was divided into $<19$ years group (children 
and adolescents) and $\geq 19$ years group (adults). Because the SEER database only records information on lymph node dissection after 1988, we only collected data on cases from 1988 to 2015 . And the years of diagnosis were divided into 1988-2005s and 2006-2015s. Tumor histological subtypes were determined according to the "ICD-O-3 Hist/Behav" field in the SEER database, We included six types of STS, the histological codes: 8890-1, 8893-6, 8900-8902, 8910, 8912, 8920, 9120, 9130, 9133, 9150, 9170, 9260, $9364,9473,8804,8005,9044$. We divided the tumor sites into three groups according to the "Primary Site - labeled" field in the SEER database. Staging information was obtained according to the SEER historic stage A (1973-2015) field. Lymph node status information was obtained from the "EOD 10-nodes (1988-2003)" and "CS-lymph nodes (2004-2015)" fields for different periods, and we divided the lymph node status into negative (N0) and positive (N1). While regional lymph node dissection information was obtained from the "Regional nodes examined (1988+)" fields. Lymph node "dissection" is defined as the removal of most or all of the nodes in the lymph node chains that drain the area around the primary tumor, include lymphadenectomy, radical node dissection, and lymph node stripping. Patients with lymph node aspiration or core biopsy, sentinel node procedure, and an uncertain number of removed lymph nodes were excluded. Based on the results of the examined lymph nodes (ELNs) data, we divided the patients into two groups: the non-NLND group and the NLND group. The tumor size data were analyzed by X-tile software (version 3.6.1) to identify the optimal tumor size cutoff value (Fig. 2), and then we divided the tumor size into three groups according to the analysis results: $<4,4-10$, and $\geq 10 \mathrm{~cm}$.

The primary outcomes of interest were patient overall survival and cancer-specific survival. Overall survival was defined as the time between first diagnosis and death of any cause, while cancer-specific survival was time from the first diagnosis to death attributed to the primary STS.

\section{Statistical Analysis}

Categorical variates are presented as frequencies and percentages, and continuous variates are presented as the median and interquartile range (IQR). For categorical variables, the Chi-square test was used to analyze between-group differences. while for continuous variables, the Wilcoxon rank-sum tests were used. For baseline variables, we performed univariate analysis by Kaplan-Meier curve and the logrank test. When the P-value was $<0.1$, the variables were further included in multivariate Cox regression analysis to identify the independent prognostic factors and estimate the hazard ratio (HR) and 95\% confidence interval $(\mathrm{Cl})$ of covariates. We identified the risk factors for LNM of STS in the head and neck and extremities by univariate and multivariate logistic regression analyses. Then, we further analyzed the effect of NLND on the prognosis of patients with STS. Taking non-cancer-specific death as a competing risk to cancer-specific death, we plotted cumulative incidence function curves for patients with NLND and performed Gray's test to compare the difference in the incidence rate of death. We performed a multivariate analysis on the cohort data to identify independent prognostic factors by using the Fine and Gray's regression model. We used the sub-distribution hazards ratio (SHR) to represent the contribution of each variable to cancer-specific death. Subsequently, we further investigated the effect of NLND on the prognosis of patients. To eliminate the impact of other factors and minimize the selection bias between the NLND and non-NLND groups, we matched patients in the NLND and non-NLND groups for propensity 
score matching (PSM) in a 1:1 ratio. We used chi-square tests to compare the clinicopathological features between the NLND and non-NLND groups, variables that differed between the two groups and those that might influence the option of treatment for negative lymph nodes were included in the matching analysis. After PSM, we performed analysis by Kaplan-Meier curve and cumulative incidence function curves. All statistical analyses in our study were performed with the R software (version 3.6.2). We use the "survival" package for the Kaplan-Meier curve and the Cox regression analysis, and the "Matchit" package was used for the propensity score matching analysis, whereas the "timereg" and "cmprsk" packages were used for the competing risk analysis. A two-sided P value of $<0.05$ was considered statistically significant.

\section{Results}

\section{Demographics and clinical characteristics of patients with STS of the head, neck and extremities}

A total of 3,276 eligible cases were included in this study. The median age (IQR range) of the entire cohort population was 53 years (31-69 years). The lymph node positivity rate for the entire cohort was $8.6 \%$ and varied significantly among tumors (Fig. 3A, $\mathrm{P}<0.001$ ). Lymph node positivity was highest for rhabdomyosarcoma (25.3\%), followed by clear cell sarcoma (16.8\%) and epithelioid sarcoma (12.4\%), and lowest for leiomyosarcoma (1.3\%). We divided the entire cohort into two groups: the negative lymph node group (NLN) and the positive lymph node group (PLN). The baseline characteristics of patients in both groups are shown in Table 1. Patients in the PLN group were significantly younger compared to the NLN group [median (IQR), 24 years ( $10-51$ years) vs. 54 years (35-70 years); $P<0.001$ ]. The tumors were mostly located in the head, neck and lower extremities in the PLN group, while in the NLN group they were mostly found in the lower extremities $(73.1 \%$ vs. $55.7 \%, P<0.001)$. Rhabdomyosarcoma was predominant in the PLN group (51.9\%), while leiomyosarcoma was predominant in the NLN group (52.7\%). The rate of NLND in the PLN group was higher ( $56.5 \%$ vs. $12.6 \% ; \mathrm{P}<0.001$ ) compared to the NLN group. There were also significant differences in race, tumor grade, stage, size, laterality, surgery and marital status between the two groups $(P<0.05)$. The median survival time in the PLN group was significantly shorter compared to the NLN group [median (IQR), 23 months (11.5-74.5 months) vs. 55 months (22-112 months); $\mathrm{P}<0.001]$.

\section{Risk factors for LNM of STS in head and neck and extremities}

Our results suggest that patients with LNM have a worse prognosis than those without LNM (Fig. 3B). Therefore, it is necessary to identify the risk factors for LNM. We performed univariate logistics and multivariate cox regression analysis analyses on the target cohort to identify risk factors for LNM. Results of univariate analysis with $P$ values less than 0.1 were included in multivariate analysis to adjust for potential confounding factors (Table 2). The result indicated that male patients [odds ratio (OR): 1.291, 
95\% Cl, 1.012-1.646; $\mathrm{P}=0.040$ ], grade III + IV (OR: 3.930, 95\% Cl, 1.805-8.554; $\mathrm{P}=0.001$ ), grade unknown (OR: 5.033, 95\%Cl, 2.325-10.895; $\mathrm{P}<0.001$ ), rhabdomyosarcoma (OR: 9.598, 95\%Cl, 5.719-16.110; $\mathrm{P}<$ 0.001), angiosarcoma (OR: 5.459, 95\% Cl, 3.163-9.419; $\mathrm{P}<0.001$ ), Ewing sarcoma (OR: 4.026, 95\% Cl, 2.205-7.351; $P<0.001$ ), epithelioid sarcoma (OR: 7.965, 95\% Cl, 4.435-14.307; $P<0.001$ ), clear cell sarcoma (OR: 11.587, 95\% Cl, 6.39-20.989; $\mathrm{P}<0.001$ ), size 4-10cm (OR: 2.080, 95\% Cl, 1.521-2.845; $\mathrm{P}<$ $0.001)$ and size $\geq 10 \mathrm{~cm}(\mathrm{OR}: 4.676,95 \% \mathrm{Cl}, 3.250-6.728 ; \mathrm{P}<0.001)$ were associated with a higher risk of LNM. while tumor site on upper limbs (OR: $0.549,95 \% \mathrm{Cl}, 0.345-0.876 ; \mathrm{P}=0.012)$ and lower limbs (OR: $0.394,95 \% \mathrm{Cl}, 0.249-0.626 ; \mathrm{P}<0.001)$ had a lower risk of LNM compared to head, face and neck site.

\section{Prognostic factors for patients with STS without LNM in the head, neck and extremities}

Of the total cohort, 2756 patients without LNM were subsequently included for survival analysis. In the Cox proportional hazards regression analysis for cancer-specific survival, patients dying of other causes are usually censured, leading to discrepancies because death for other factors will prevent the occurrence of target events. Considering death for other causes as the competing risk, we further conducted Fine and Gray's regression analysis (Table 3). The results of the analysis showed that factors associated with cancer-specific survival after controlling for competing risks were age, histology, tumor size, grade, stage, and marital status. Age $\geq 19$ years (sHR, 1.876, 95\% Cl, 1.341-2.625; $\mathrm{P}<0.001)$, grade III + IV (sHR: 1.771, $95 \% \mathrm{Cl}, 1.422-2.206 ; \mathrm{P}<0.001)$, Other (sHR: $1.400,95 \% \mathrm{Cl}, 1.100-1.783 ; \mathrm{P}=0.01$ ), rhabdomyosarcoma (sHR: 1.387, 95\% Cl, 1.080-1.779; P = 0.01), angiosarcoma (sHR: 1.817, 95\% Cl, 1.477-2.237; $\mathrm{P}<0.001$ ), epithelioid sarcoma (sHR: 1.463, 95\% Cl, 1.029-2.080; $\mathrm{P}=0.03$ ), clear cell sarcoma (sHR: $2.321,95 \% \mathrm{Cl}$, 1.616-3.333; $\mathrm{P}<0.001)$, stage of regional (sHR, 1.452, 95\% Cl, 1.220-1.728; $\mathrm{P}<0.001)$, distant (sHR, 4.428, 95\% Cl, 3.356-5.843; $\mathrm{P}<0.001$ ), size 4-10cm (sHR: 2.655, 95\% Cl, 2.174-3.243; $\mathrm{P}<0.001$ ) and size $\geq 10 \mathrm{~cm}$ (sHR: 4.656, 95\% Cl, 3.699-5.861; P<0.001), Divorced/Widowed/Separated (sHR: 1.554, $95 \% \mathrm{Cl}, 1.272-1.897 ; \mathrm{P}<0.001)$ were associated with an increased risk of cancer-specific death. But NLND was not associated with cancer-specific mortality (sHR: $0.838,95 \% \mathrm{Cl}, 0.662-1.061 ; \mathrm{P}=0.14$ ). The remaining factors such as sex, race, year of diagnosis, site and laterality had no statistical effect on cancer-specific death.

\section{The effect of NLND on the prognosis of patients with different soft tissue sarcomas}

We further analyzed the effect of NLND on the prognosis of patients for different histological subtypes of STS. We found that NLND was an independent risk factor for the prognosis of five subtypes of STS at high risk for LNM: rhabdomyosarcomas, angiosarcomas, Ewing sarcomas, epithelioid sarcomas, and clear cell sarcomas. NLND group had significantly higher overall and cancer-specific survival rates (Fig. 4A-B). We also plotted cumulative incidence function curves to compare the differences in cancerspecific mortality between the two groups. As shown in Fig. 6A, the cumulative incidence of cancerspecific death in the NLND group was lower than that in the non-NLND group (Gray's test, $P=0.001$ ). However, NLND does not improve cancer-specific survival in patients with leiomyosarcoma (Fig. 5B). To 
further clarify the effect of NLND on patients' prognosis, PSM analysis was performed. First of all, we used chi-square tests to compare the baseline characteristics between the NLND and non-NLND groups, The results showed that there were significant differences between the two groups for age, site, laterality, histology, stage, size and marital status ( $p<0.05$, Table 4$)$. Then, we match these covariables to get a new cohort. After PSM, the covariates between the two groups were balanced. We analyze the cohort data after PSM, and the results further confirmed that NLND significantly improved overall survival (HR: 0.718 , $95 \% \mathrm{Cl}, 0.535-0.962 ; \mathrm{P}=0.026)$ and cancer-specific survival (HR: $0.699,95 \% \mathrm{Cl}, 0.506-0.967 ; \mathrm{P}=0.031$ ), as detailed in Table 5 and Fig. 4C-D. And as shown in Fig. 6B, patients in the NLND group had significantly lower cancer-specific mortality (Gray's test, $P=0.017$ ). Moreover, patients in the NLND group had a significantly longer median survival time compared to patients in the non-NLND group, before (median: 69 months vs 47 months, respectively; $p$ < 0.001) and after PSM (median: 69.5 months vs 57.5 months, respectively; $p=0.080$ ). However, for patients with leiomyosarcoma, NLND did not improve overall survival $(P=0.46)$ or reduce cancer-specific mortality (Gray's test, $P=0.772)$, as detailed in Fig. $5 C$ D and Fig. 6D.

\section{Discussion}

The prognosis of STS with metastasis is poor, and the main metastasis sites of STS are lung and bone, LNM is relatively rare. The latest AJCC staging system defines lymph node involvement as being stage IV disease for sarcomas of the trunk and extremities[1]. This indicates that LNM is a significant prognostic factor. Therefore, it is necessary to study the risk factors and prognosis of LNM in STS. At present, most studies have included all types of STS or site-specific, patient-specific STS. For example, Gusho et al. studied the LNM rate and prognosis of all STS of the extremities[11]. Sherman et al. studied the LNM rate and predictors of adult STS of extremities. Our study included six types of STS with the highest risk of LNM in the head, neck and extremities. We analyzed the prognosis of 2,756 patients with STS without LNM and who were treated surgically. Also, we further clarified the impact of NLND on the prognosis of these patients.

In our cohort, rhabdomyosarcoma (25.3\%), clear cell sarcoma (16.8\%) and epithelioid sarcoma $(12.4 \%)$ had the highest rates of LNM, while leiomyosarcoma had the lowest rate of LNM at $1.3 \%$. This is consistent with previously reported rates of lymph node positive of different STS (26.7\% for rhabdomyosarcoma, $16-18.8 \%$ for clear cell sarcoma, and $13-14.5 \%$ for epithelioid sarcoma) from several large cohort studies $[8,11,13]$. In children and adolescents ( $<19$ years), rhabdomyosarcoma has the highest positive rate of lymph node, while in adults ( $\geq 19$ years) is clear cell sarcoma. The results are similar to previous studies[8, 14]. This may be related to differences in the histological subtypes of STS in different populations of age, rhabdomyosarcoma was the most common STS in children and adolescents and accounts for one-half of pediatric STS[15]. We identified independent risk factors for LNM using the multivariate Cox proportional risk model. Male, head and neck, high grade (III + IV), tumor size greater than $4 \mathrm{~cm}$, non-leiomyosarcomas are more likely to have LNM. Several studies have reported similar results. Miccio et al. found that high-grade and clear cell/ angiosarcoma/ rhabdomyosarcoma/ epithelioid (CARE) histology are associated with LNM in STS[7]. In another study, Behranwala et al. 
examined 2,127 STS, finding a 70\% association between high-grade tumors and lymph nodes spread, and LNM are more likely to appear in the proximal location of the sarcoma[6]. Sherman et al. included 27,536 patients of extremity soft tissue sarcoma (ESTS) from the National Cancer Data Base (20002009) and found that the risk factors for LNM were histologic subtype, tumor size, and grade[14]. In addition, some studies have shown that LNM is also related to age and primary site[10, 16]. However, no studies have reported an association between gender and LNM. Our results suggest that male is at higher risk of LNM compared to female (OR: 1.291, 95\% Cl, 1.012-1.646; $\mathrm{P}=0.040$ ). The literature reports that males have historically been associated with a higher predisposition to STS than females, but the extent to which gender affects lymph node metastasis has not been established [17].

We performed a prognostic analysis of 2,756 patients without LNM and who had undergone surgery. Age, grade, stage, size, histology and marital status were found to be independent prognostic factors for cancer-specific survival. This result is similar to previous studies. A study with patient data also from the SEER database showed that for the historically high-risk extremity STS, age, grade, size, surgery, and regional lymph node status were independent disease-specific prognostic factors[11]. Another study found that for epithelial sarcoma, tumor site was a prognostic factor for event-free survival and overall survival, and extremities site had a better prognosis than proximal-type variant[18]. Understanding these characteristics of STS can help us to better provide clinical counseling and personalized treatment for patients.

The current study further investigated the association between NLND and prognosis. After PSM, we found that NLND was an independent prognosis factor for patients with a high risk of LNM such as rhabdomyosarcomas, angiosarcomas, Ewing sarcomas, epithelioid sarcomas, and clear cell sarcomas. Surprisingly, NLND can not improve the prognosis of leiomyosarcoma before and after PSM. We further analyzed the leiomyosarcoma cohort and found that most of the leiomyosarcoma patients were older than the rest five types of sarcomas (median age: 61 years vs. 41 years, $\mathrm{P}<0.001$ ) and only a small proportion of the population (6.81\%) received NLND compared with $21.2 \%$ of other tumors. Moreover, we can't get information from the SEER database of the reasons why these patients did not have lymph node dissection. It may be that these patients are older, have comorbidities or have treatment contraindications. And the SEER database did not record the treatment information of these patients about adjuvant treatments such as radiotherapy and chemotherapy. Above all of these factors may affect the accuracy of the results and cause confounding bias. Therefore, further clinical validation is needed.

At present, some studies have reported the prognosis of lymph node examination/dissection for STS with LNM, but the result remains controversial. Al-Refaie et al suggest that regional lymph node dissection may prolong survival time[19]. Ecker et al support regional lymph node examination for patients with epithelioid and possibly clear cell sarcoma[20]. Brady et al found that lymph node sampling was associated with improved disease-specific survival in patients with extremity rhabdomyosarcoma (64\% versus $49 \%, \mathrm{P}=0.005$ ) [21]. Riad et al show that resection of involved lymph nodes had an estimated 5year survival of $57 \%$, whereas nine patients treated without surgery all died within 30 months[22]. In 
another study, NLND was proved to be an independent risk factor for cancer-specific survival in nonmetastatic colorectal sarcomas patients[23]. However, some studies have found that lymph node examination/dissection has no effect on prognosis. A study of epithelioid sarcoma found that lymphadenectomy did not improve overall survival in patients with LNM[24]. Another study found that resection of the metastatic lymph node had better survival at 1.5 years, but did not improve the long-term survival of patients with STS[9]. Some studies think the management of positive lymph nodes remains uncertain, and the effect of lymphadenectomy on the overall survival of STS with LNM needs to be further clarified $[3,10]$. In summary, our study suggests that NLND is appropriate treatment for specific patients with STS, such as those who have a high risk of LNM. Nevertheless, we still recommend that patients' treatment decisions should be based on the clinical reality of the patient, because lymph node dissection may have some acute and chronic complications, such as lymphorrhea, chylous ascites, seroma, delayed wound healing and chronic lymphedema[25].

Our study has some limitations which need to be considered. First, this is a retrospective study and may have inherent limitations, so our results must be validated in prospective studies. Second, the SEER database does not record detailed information about chemotherapy, radiotherapy, comorbidities, complications, and recurrence, which may have a potential impact on the results. Despite these limitations, our findings are of significance.

\section{Conclusion}

Our study identified the rate of LNM in six subtypes STS of the head, neck, and extremites. In addition, we further clarified the risk factors for LNM and the prognostic factors for patients with STS without LNM. Most importantly, our study suggests that prophylactic lymph node dissection is necessary and clinically beneficial for STS with a high risk of LNM in the head, neck and extremities. However, for leiomyosarcoma, NLND does not improve the prognosis and prophylactic lymph node dissection needs to be evaluated carefully.

\section{Abbreviations}

LNM

lymph node metastasis; STS:soft tissue sarcomas; NLND:negative lymph node dissection; SEER:the Surveillance, Epidemiology, and End Results database; NLN:lymph node group; PLN:positive lymph node group; PSM:propensity score matching; IQR:interquartile range; HR:hazard ratio; Cl:confidence interval; OR:odds ratio; CARE:clear cell/ angiosarcoma/ rhabdomyosarcoma/ epithelioid; ESTS:extremity soft tissue sarcoma

\section{Declarations}

\section{Funding}


The study was funded by National Natural Science Foundation of China (No. 81472106).

\section{Author contributions}

(I) Conception and design: Hao Kang; (II) Collection and assembly of data: Mengwei Li; Zhiwei Li; Yongqiao Jiang; (III) Data analysis and interpretation: Qikun Liu; (IV) Manuscript review and revision: Xiaojun Yu; (V) Manuscript writing: All authors; (VI) Final approval of manuscript: All authors.

\section{Ethics approval and consent to participate}

The raw data of this study are derived from the SEER database (https://seer.cancer.gov/), which is a publicly available database. And individual consent for this retrospective analysis was waived as the patient information is anonymous.

\section{Consent for publication}

Not applicable

\section{Conflict of interest}

The authors declare no conflict of interest.

\section{Acknowledgments}

We wish to thank the Surveillance, Epidemiology, and End Results (SEER) Program.

\section{Availability of data and materials}

All detailed data included in the study are available upon request by contact with the corresponding author (SEER ID:14648-Nov2019).

\section{References}

1. von Mehren M, Randall RL, Benjamin RS, Boles S, Bui MM, Ganjoo KN, George S, Gonzalez RJ, Heslin MJ, Kane JM, et al. Soft Tissue Sarcoma, Version 2.2018, NCCN Clinical Practice Guidelines in Oncology. J Natl Compr Canc Netw. 2018;16:536-63.

2. Gutierrez JC, Perez EA, Franceschi D, Moffat FL Jr, Livingstone AS, Koniaris LG. Outcomes for softtissue sarcoma in 8249 cases from a large state cancer registry. J Surg Res. 2007;141:105-14.

3. Jacobs AJ, Morris CD, Levin AS. Synovial Sarcoma Is Not Associated With a Higher Risk of Lymph Node Metastasis Compared With Other Soft Tissue Sarcomas. Clin Orthop Relat Res. 2018;476:58998.

4. Kim JR, Yoon HM, Koh KN, Jung AY, Cho YA, Lee JS. Rhabdomyosarcoma in Children and Adolescents: Patterns and Risk Factors of Distant Metastasis. AJR Am J Roentgenol. 2017;209:40916. 
5. Nishida Y, Tsukushi S, Urakawa H, Sugiura H, Nakashima H, Yamada Y, Ishiguro N. High incidence of regional and in-transit lymph node metastasis in patients with alveolar rhabdomyosarcoma. Int $\mathrm{J}$ Clin Oncol. 2014;19:536-43.

6. Behranwala KA, A'Hern R, Omar AM, Thomas JM. Prognosis of lymph node metastasis in soft tissue sarcoma. Ann Surg Oncol. 2004;11:714-9.

7. Miccio JA, Jairam V, Gao S, Augustyn A, Oladeru OT, Onderdonk BE, Chowdhary M, Han D, Khan S, Friedlaender G, et al. Predictors of Lymph Node Involvement by Soft Tissue Sarcoma of the Trunk and Extremity: An Analysis of the National Cancer Database. Cureus. 2019;11:e6038.

8. Keung EZ, Chiang YJ, Voss RK, Cormier JN, Torres KE, Hunt KK, Feig BW, Roland CL. Defining the incidence and clinical significance of lymph node metastasis in soft tissue sarcoma. Eur $\mathrm{J}$ Surg Oncol. 2018;44:170-7.

9. Sawamura C, Matsumoto S, Shimoji T, Ae K, Okawa A. Lymphadenectomy and histologic subtype affect overall survival of soft tissue sarcoma patients with nodal metastases. Clin Orthop Relat Res. 2013;471:926-31.

10. Johannesmeyer D, Smith V, Cole DJ, Esnaola NF, Camp ER. The impact of lymph node disease in extremity soft-tissue sarcomas: a population-based analysis. Am J Surg. 2013;206:289-95.

11. Gusho CA, Fice MP, O'Donoghue CM, Gitelis S, Blank AT. A Population-based Analysis of Lymph Node Metastasis in Extremity Soft Tissue Sarcoma: An Update. J Surg Res. 2021;262:121-9.

12. Crettenand F, Martin D, Cherix S, Demartines N, Matter M. Occurrence and prognosis of lymph node metastases in patients selected for isolated limb perfusion with soft tissue sarcoma. $\mathrm{J}$ Cancer. 2018;9:3311-5.

13. Daigeler A, Kuhnen C, Moritz R, Stricker I, Goertz O, Tilkorn D, Steinstraesser L, Steinau HU, Lehnhardt M. Lymph node metastases in soft tissue sarcomas: a single center analysis of 1,597 patients. Langenbecks Arch Surg. 2009;394:321-9.

14. Sherman KL, Kinnier CV, Farina DA, Wayne JD, Laskin WB, Agulnik M, Attar S, Hayes JP, Peabody T, Bilimoria KY. Examination of national lymph node evaluation practices for adult extremity soft tissue sarcoma. J Surg Oncol. 2014;110:682-8.

15. Amer KM, Thomson JE, Congiusta D, Dobitsch A, Chaudhry A, Li M, Chaudhry A, Bozzo A, Siracuse B, Aytekin MN, et al. Epidemiology, Incidence, and Survival of Rhabdomyosarcoma Subtypes: SEER and ICES Database Analysis. J Orthop Res. 2019;37:2226-30.

16. Li RH, Zhou Q, Li AB, Zhang HZ, Lin ZQ. A nomogram to predict metastasis of soft tissue sarcoma of the extremities. Med (Baltim). 2020;99:e20165.

17. Rosenthal J, Cardona K, Sayyid SK, Perricone AJ, Reimer N, Monson D, Singer AD. Nodal metastases of soft tissue sarcomas: risk factors, imaging findings, and implications. Skeletal Radiol. 2020;49:221-9.

18. Casanova M, Ferrari A, Collini P, Bisogno G, Alaggio R, Cecchetto G, Gronchi A, Meazza C, Garaventa A, Di Cataldo A, et al. Epithelioid sarcoma in children and adolescents: a report from the Italian Soft Tissue Sarcoma Committee. Cancer. 2006;106:708-17. 
19. Al-Refaie WB, Andtbacka RH, Ensor J, Pisters PW, Ellis TL, Shrout A, Hunt KK, Cormier JN, Pollock RE, Feig BW. Lymphadenectomy for isolated lymph node metastasis from extremity soft-tissue sarcomas. Cancer. 2008;112:1821-6.

20. Ecker BL, Peters MG, McMillan MT, Sinnamon AJ, Zhang PJ, Kelz RR, Roses RE, Drebin JA, Fraker DL, Karakousis GC. Implications of Lymph Node Evaluation in the Management of Resectable Soft Tissue Sarcoma. Ann Surg Oncol. 2017;24:425-33.

21. Brady AC, Picado O, Tashiro J, Sola JE, Perez EA. Lymph Node Sampling and Survival in Child and Adolescent Extremity Soft-Tissue Sarcoma. J Surg Res. 2019;241:205-14.

22. Riad S, Griffin AM, Liberman B, Blackstein ME, Catton CN, Kandel RA, O'Sullivan B, White LM, Bell RS, Ferguson PC, Wunder JS. Lymph node metastasis in soft tissue sarcoma in an extremity. Clin Orthop Relat Res 2004:129-134.

23. Wei Z, Mao R, Zhang Y, Bi X, Zhou J, Li Z, Huang Z, Chen X, Zhao J, Zhao H, Cai J. The prognostic factors of primary colorectal sarcoma and the clinical outcomes of negative lymph node dissection. Ann Transl Med. 2021;9:250.

24. Outani H, Imura Y, Tanaka T, Takenaka S, Oshima K, Hamada K, Kakunaga S, Joyama S, Naka N, Kudawara I, et al. Clinical outcomes of patients with epithelioid sarcomas: impact and management of nodal metastasis. Int J Clin Oncol. 2018;23:181-8.

25. Morton DL, Cochran AJ, Thompson JF, Elashoff R, Essner R, Glass EC, Mozzillo N, Nieweg OE, Roses $D F$, Hoekstra HJ, et al. Sentinel node biopsy for early-stage melanoma: accuracy and morbidity in MSLT-I, an international multicenter trial. Ann Surg. 2005;242:302-11. discussion 311 - 303.

\section{Tables}

Due to technical limitations, table 1 to 5 is only available as a download in the Supplemental Files section.

\section{Figures}


Patients diagnosed with soft tissue sarcoma of head and neck and extremities

(HNESTS) in the SEER database from 1988 to 2015 ( $N=34297$ )

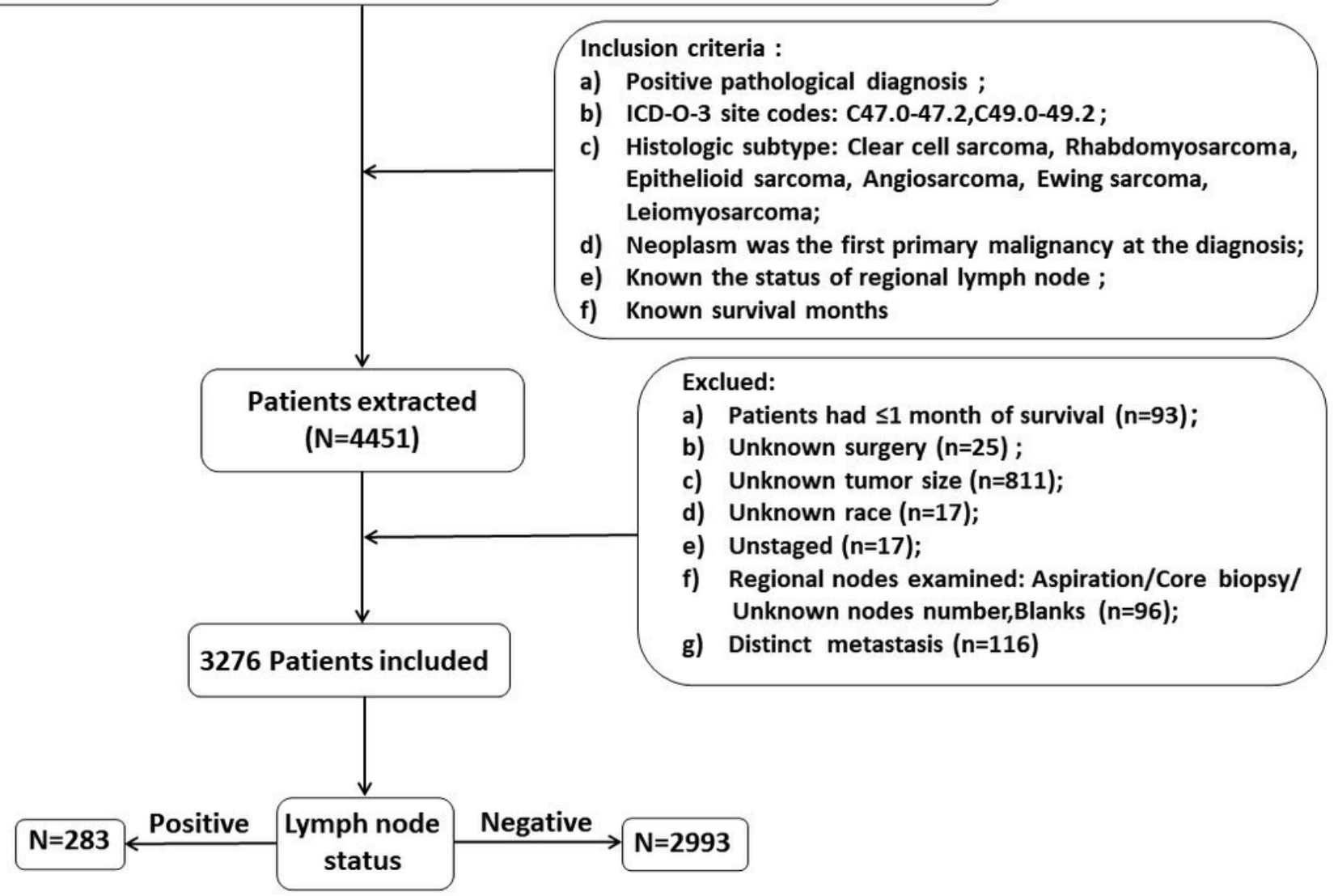

Figure 1

Flow chart of the selection of patients with STS of the head, neck and extremities from the Surveillance, Epidemiology, and End Results database. 



D

\section{Figure 2}

The optimal tumor size cut-off values according to cancer-specific survival by X-tile analysis. X-tile software was used to calculate the cut-off value of tumor size (A and B). The cohort was divided into low (purple), medium (grey) and high (blue) survival groups according to the cut-off value for tumor size (C). The cut-off value for tumor size was validated by Kaplan-Meier curve (D). 

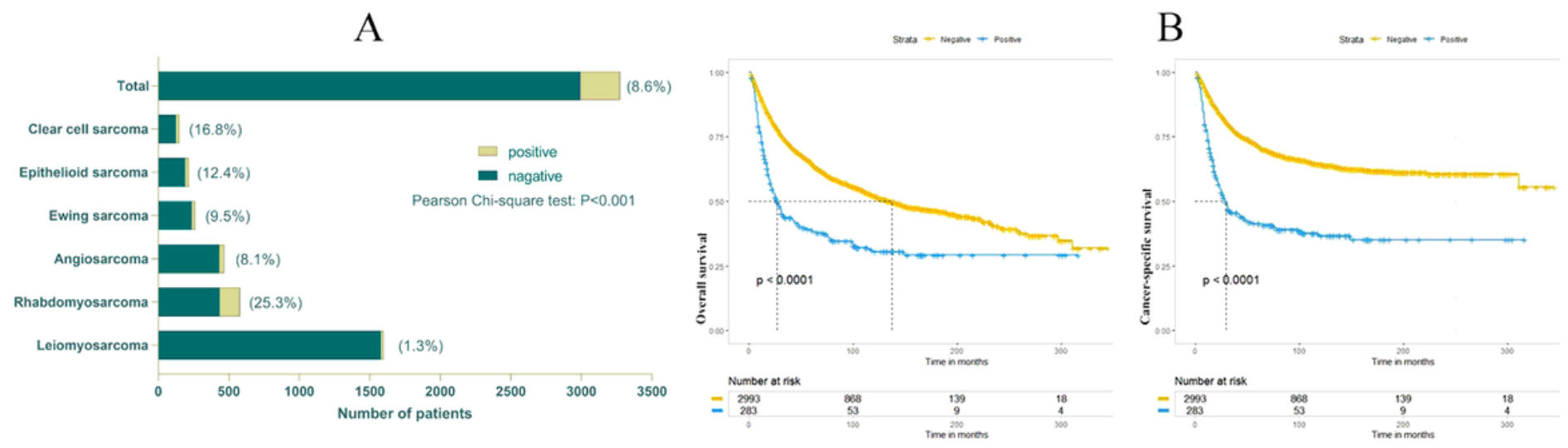

Figure 3

LNM rates in different histological subtypes of STS and the Kaplan-Meier survival curve analysis of the impact of LNM on the prognosis of STS: A. results of LNM rates for different tumors; B. overall survival and cancer-specific survival of LNM. 
A
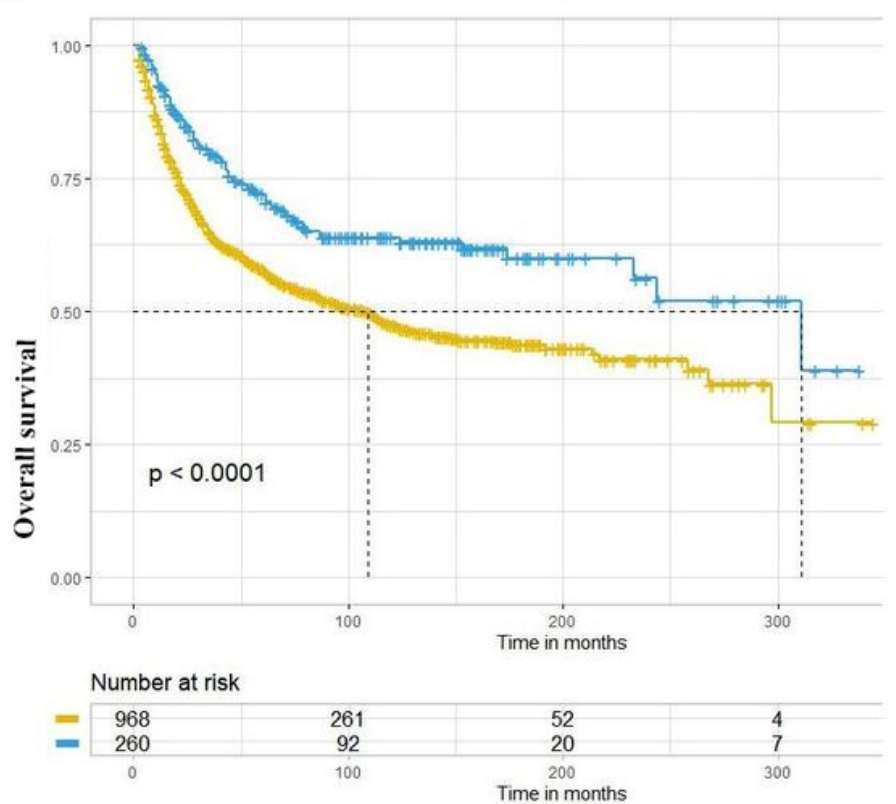

C

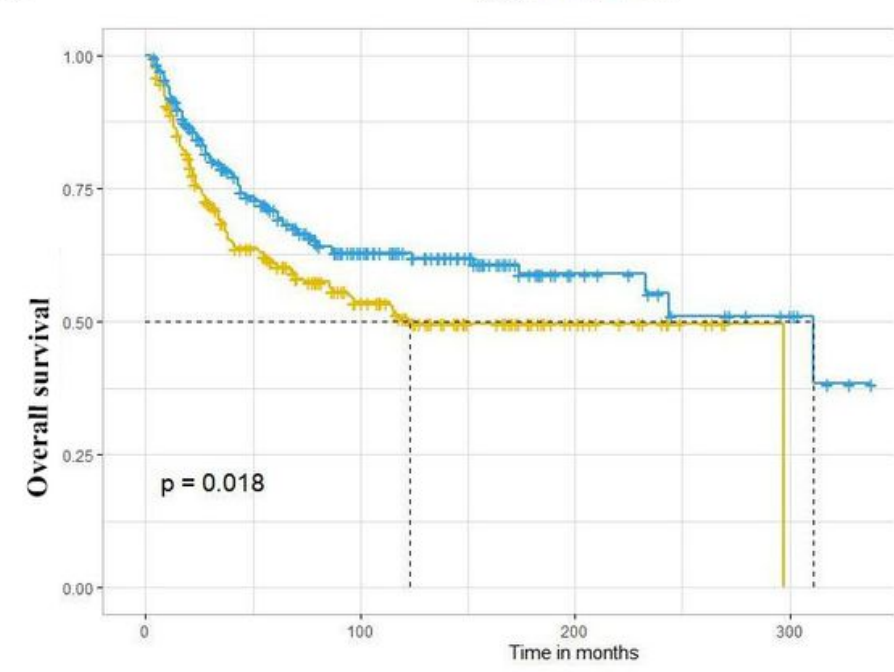

Number at risk

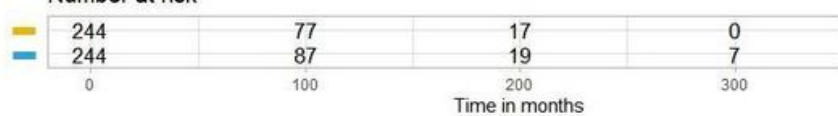

B
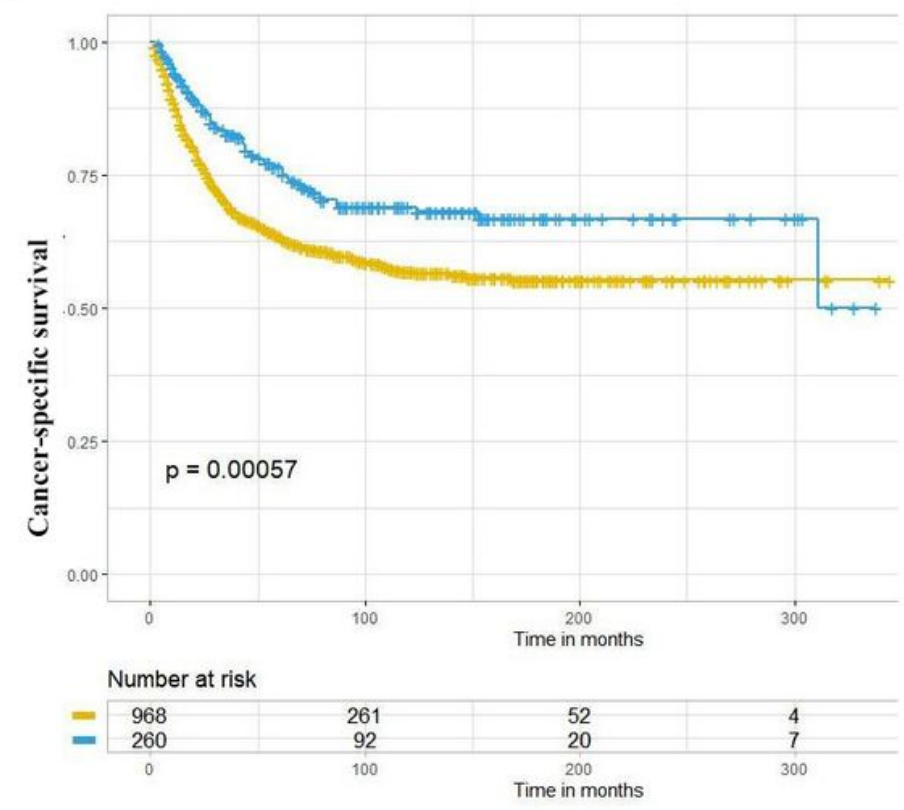

D
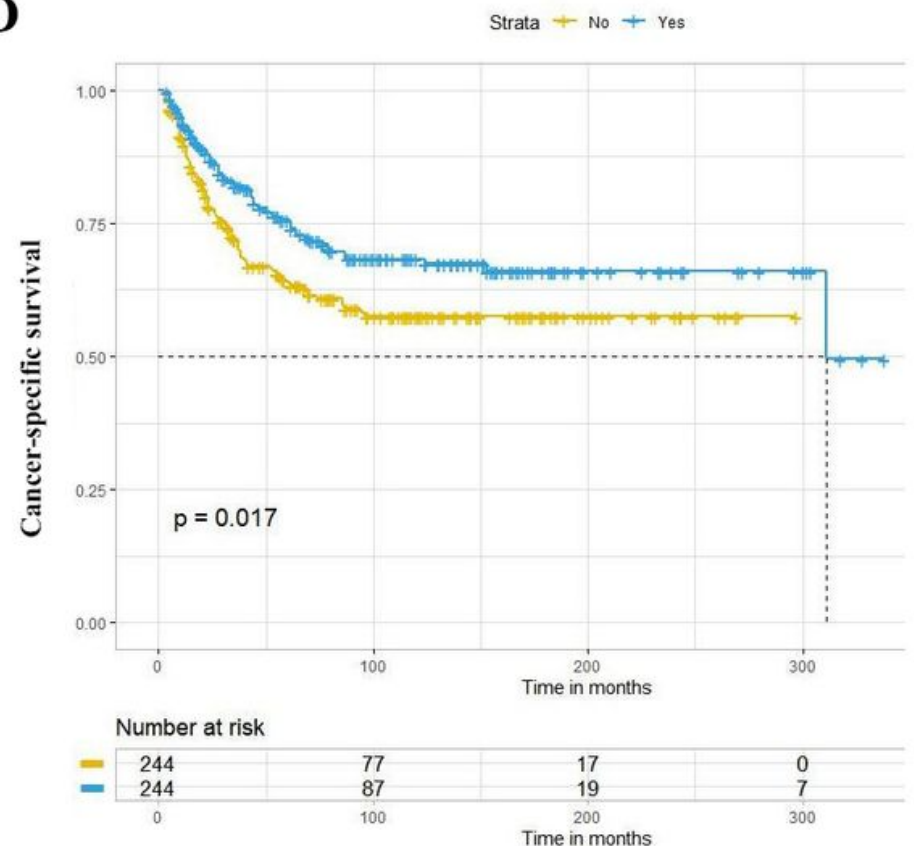

\section{Figure 4}

Kaplan-Meier survival curves for overall survival and cancer-specific survival of five subtypes STS with a high risk of LNM between the NLND group and non-NLND group : A-B in the raw cohort and C-D in the PSM cohort. 
A
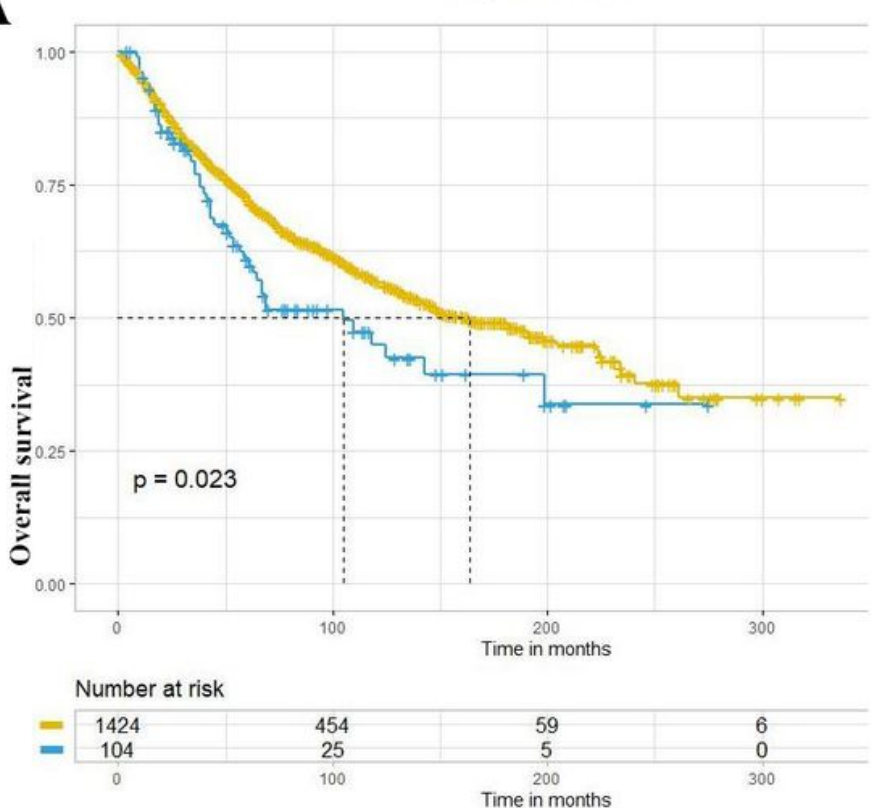

C
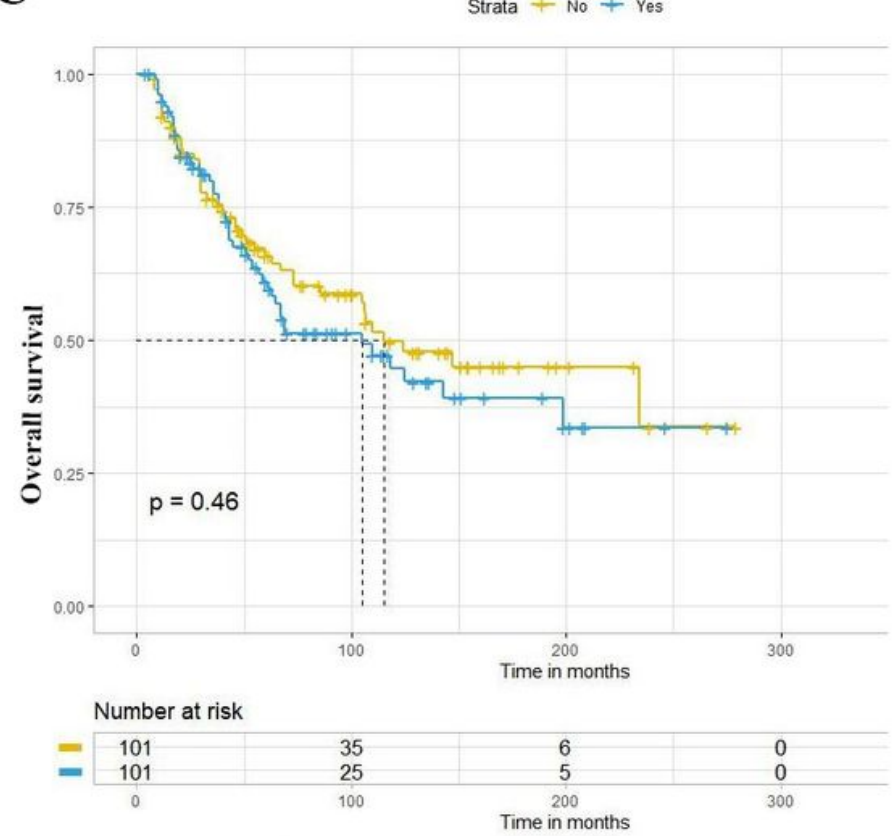

B
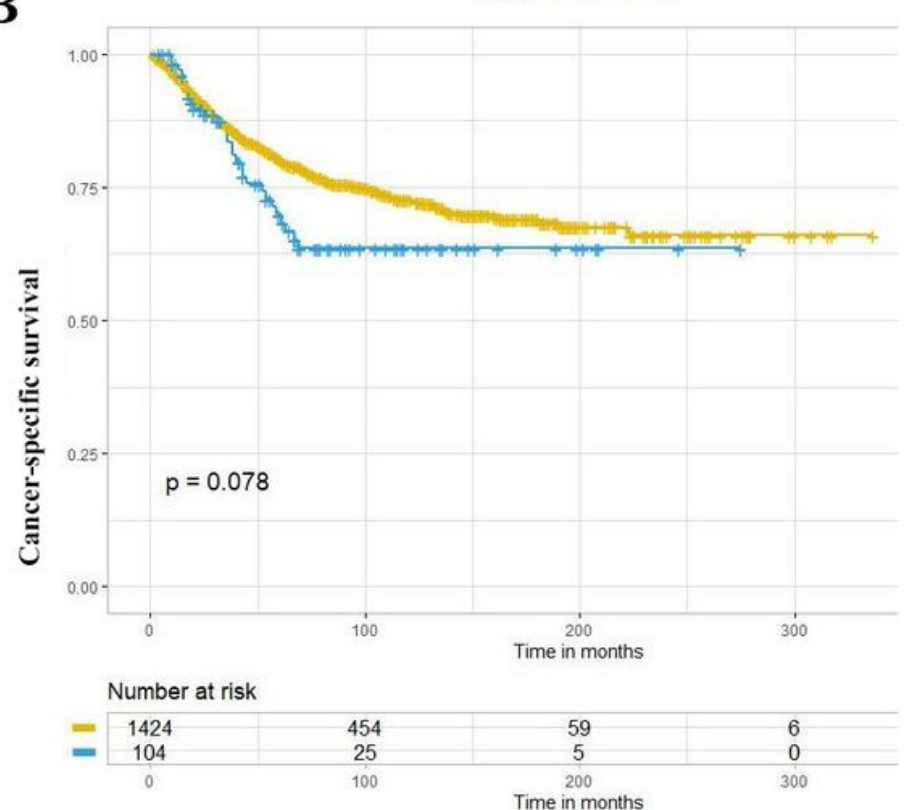

D
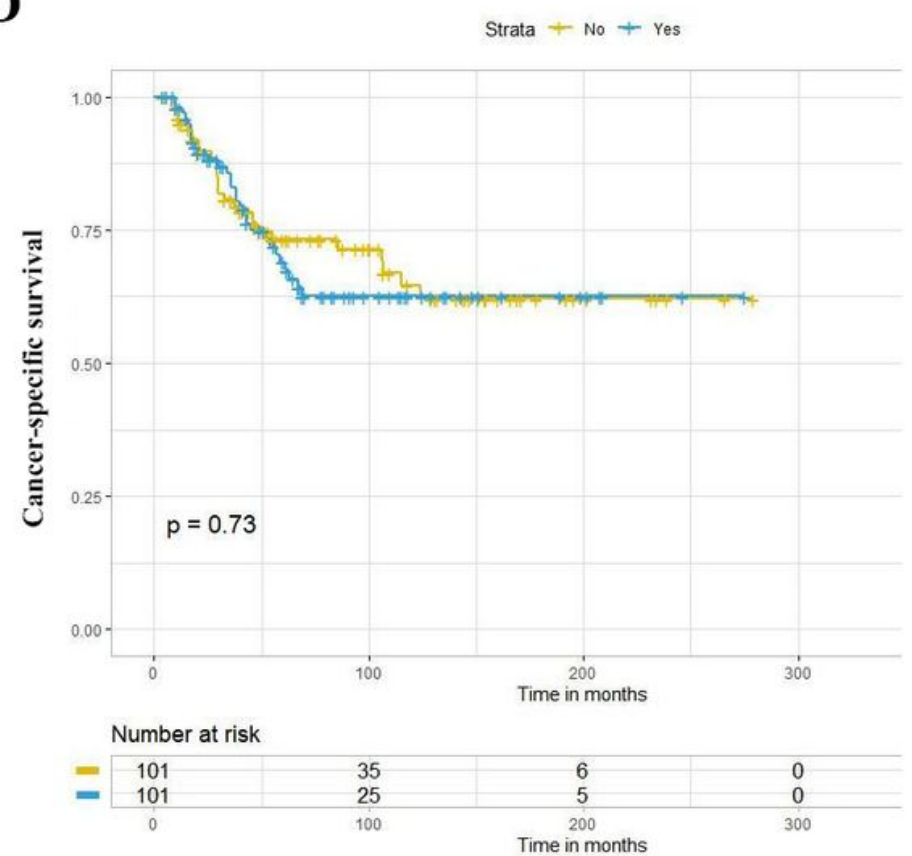

\section{Figure 5}

Kaplan-Meier survival curves for overall survival and cancer-specific survival between the NLND group and non-NLND group with leiomyosarcoma: A-B in the raw cohort and C-D in the PSM cohort. 

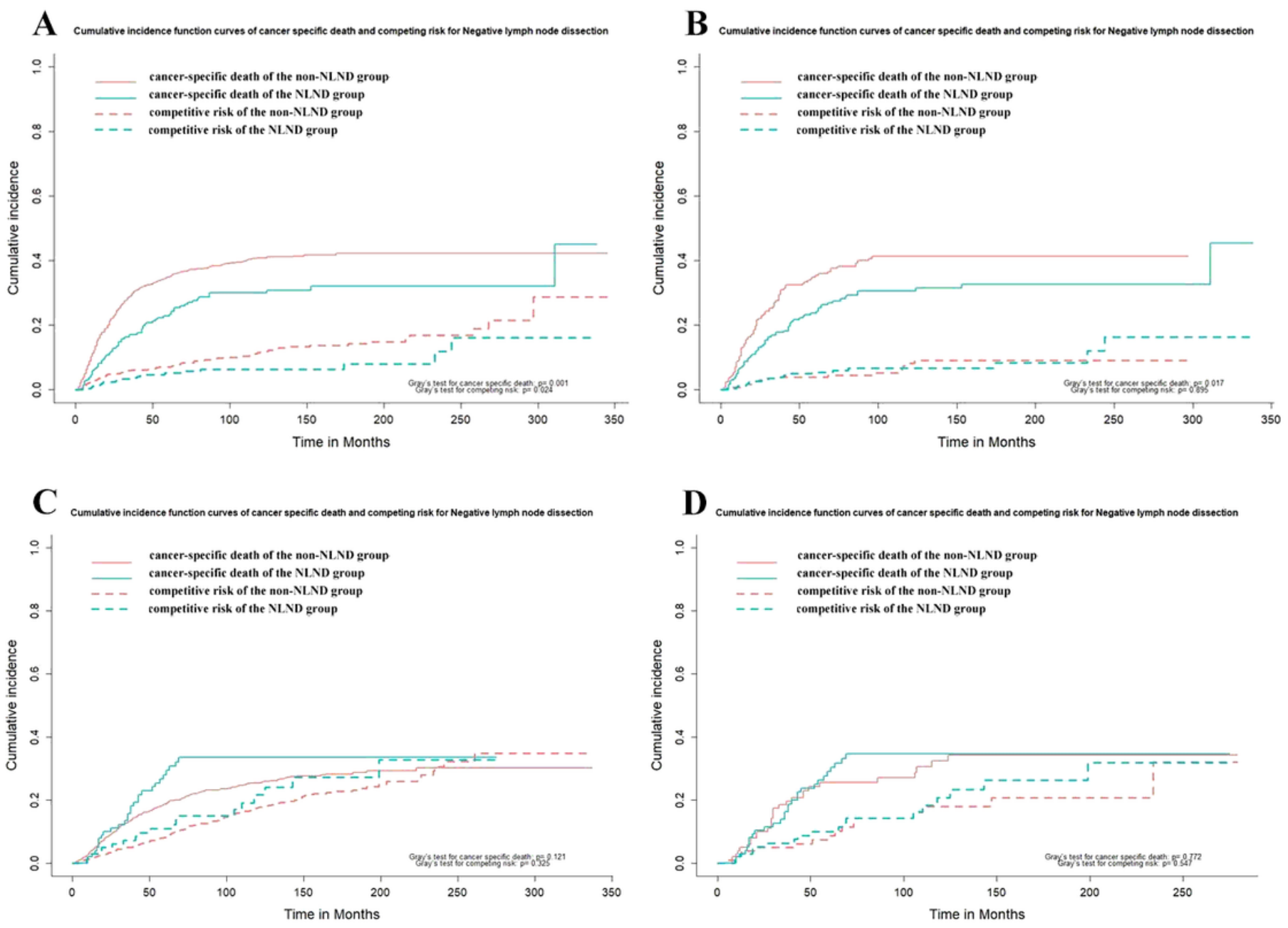

Figure 6

The cumulative incidence of cancer-specific death and competitive risk between the NLND group and non-NLND group of five subtypes STS with a high risk of LNM (A-B) or leiomyosarcoma (C-D): A /C in the raw cohort and $B / D$ in the PSM cohort.

\section{Supplementary Files}

This is a list of supplementary files associated with this preprint. Click to download.

- Table1.xlsx

- Table2.xlsx

- Table3.xlsx

- Table4.xlsx

- Table5.xlsx 